\title{
¿Cuál es el nivel de desarrollo esperable para un niño con síndrome de Down en sus primeros años de vida?*
}

\section{What is the Level of Development Expected for a Child with Down syndrome in their First Years of Life?}

\author{
Juan Carlos Rodríguez-Barrera a \\ Universidad de Antioquia, Colombia \\ ORCID: http://orcid.org/0000-0003-2776-0539 \\ Liliana Chaves-Castaño \\ Universidad de Antioquia, Colombia
}

\footnotetext{
a Autor de correspondencia. Correo electrónico: juancarlosrodriguez.001@gmail.com
}

Para citar este artículo: Rodríguez-Barrera, J., \& Chaves-Castaño, L. (2017). ¿Cuál es el nivel de desarrollo esperable para un niño con síndrome de Down en sus primeros años de vida? Universitas Psychologica, 16(4), 1-13. https://doi.org/10.11144/Jav eriana.upsy16-4.cnde

\section{RESUMEN}

El síndrome de Down es la causa genética más frecuente de discapacidad vinculada con el desarrollo. La falta de valores normativos para los niños que padecen este trastorno, dificulta una valoración temprana y seguimiento del curso evolutivo. El objetivo del presente artículo es describir el perfil evolutivo de estos niños durante los primeros tres años de vida. Se realizó un estudio de cohorte de tipo prospectivo durante 9 años, en el cual se incluyeron 156 niños menores de 37 meses. Ellos tuvieron 528 evaluaciones con la "Escala Abreviada del Desarrollo" (EAD-1), una prueba válida y confiable estandarizada en Colombia para valorar el nivel evolutivo en niños menores de 6 años. Se establecieron los cocientes de desarrollo por trimestres, valores percentil, las variaciones entre las áreas evaluadas, las curvas evolutivas estudiadas en cada área y los períodos más críticos en los diferentes semestres. Estos valores posibilitarán el seguimiento al curso evolutivo de estos niños, la identificación temprana de las áreas de mayor vulnerabilidad y necesidad de intervención, la evaluación del impacto de acciones terapéuticas o programas de estimulación, y la orientación a los padres y agentes sociales y de salud vinculados al desarrollo y la supervivencia del niño y la niña.

\section{Palabras clave}

síndrome de Down y desarrollo; cocientes de desarrollo; discapacidad infantil; escala abreviada del desarrollo-EAD-1.

\begin{abstract}
Down syndrome is the most frequent genetic cause of disability associated with development. The lack of normative values for these children, hinders an early assessment and monitoring of the clinical course. The objective was to describe the evolutionary profile of these children during the first three years of life. A prospective cohort study was performed, for 9 years, in which 156 children under 37 months were included. They had 528 evaluations by "Abbreviated Scale Development" (EAD -1), a valid and reliable standardized test in Colombia to assess the developmental level in children under 6 years. We obtained quarterly developmental quotients, percentile values, variations between the areas assessed, the evolutionary curve in each area studied and the most critical
\end{abstract}


periods in different semesters. These values will enable monitoring the clinical course of these children, early identification of areas of greatest vulnerability and need for intervention, assessment of the impact of therapeutic interventions or programs of stimulation and guidance to parents and related health and social agents to development and survival of the boy and the girl.

Keywords

developmental; Down syndrome and neurodevelopment; developmental quotient; child and handicap; abbreviated scale development EAD-1.

El síndrome de Down (SD) o trisomía 21 es una condición genética producida por una falta de disyunción o una translocación del cromosoma 21. La prevalencia de este síndrome en todo el mundo es de alrededor de 1 por cada 700 nacimientos (Hayes \& Batshaw, 1993; Martin, Klusek, Estigarribia, \& Roberts, 2005). En Colombia se estima que cada año nacen cerca de 1000 niños con el síndrome (Rodríguez, 2012). Es la afección más antigua asociada con discapacidad intelectual y la causa genética más frecuente relacionada con alteraciones en el desarrollo (Abbeduto, Warren, \& Conners, 2007; Antonarakis, Lyle, Chrast, \& Scott, 2001; Pinter, Eliez, Schmitt, Capone, \& Reiss, 2001). Estas alteraciones están asociadas en gran medida a la sobrecarga genética aportada por el cromosoma 21 extra, ya que origina un desequilibrio en los procesos que regulan la embriogénesis y en particular del sistema nervioso central (Flórez, 1991; Rodríguez 2012). Por lo tanto, esta anomalía cromosómica converge, en la mayoría de los casos, en un retardo mental o discapacidad intelectual.

Si bien esta discapacidad debe ser diagnosticada luego de los primeros cinco años de vida por medio del coeficiente intelectual (IQ), las tempranas evaluaciones del desarrollo infantil revisten un gran valor al ayudar a detectar e intervenir oportunamente retrasos o alteraciones que son determinantes para el funcionamiento intelectual posterior (Rodríguez, 2005). Los test de desarrollo evalúan, en su gran mayoría, la organización progresiva de funciones básicas como: la actividad motora y sensorial, el lenguaje, la interacción social, e incluso la atención y la memoria (Moore, Goodwin, \& Oates, 2008). En el caso de los niños con SD, todas estas funciones tienden a estar afectadas, condición que contribuye a la discapacidad cognitiva posterior.

No todos los niños con SD nacen con una patología igualmente intensa, ni siguen el mismo proceso de desarrollo a lo largo de sus vidas (Artigas, 2004). Entonces, icómo saber los efectos de esa sobrecarga génica en las capacidades de estos niños? Un indicador del nivel evolutivo alcanzado por un niño es el cociente de desarrollo (CD) obtenido por los test de desarrollo, el cual expresa una relación entre la edad madurativa del niño y su edad cronológica. Entre más lejos esté la edad madurativa de la cronológica, mayor es el grado de afectación que tiene el niño en su neurodesarrollo. La mayoría estos test evalúan cuatro áreas: motricidad gruesa, motricidad fina, lenguaje y la personal y social.

Si bien existen estudios respecto a los (IQ) en jóvenes y adultos con $\mathrm{SD}$, son pocas las investigaciones que indiquen claramente los $C D$ en estos niños durante los primeros años de vida. En general, estos estudios son transversales, y agrupan niños de diferentes edades como lactante, preescolares y escolares y promediando sus resultados, o se enfocan en una sola función. Por ejemplo, en 1978 Chen y Woolley usaron el Denver Developmental Screening Test en 106 niños con SD con edades entre los dos meses y los ocho años (Palisano et al., 2001). Centrados en una sola área del desarrollo, Palisano y colaboradores (2001), evaluaron el desarrollo motor de 121 niños con SD, con edades entre uno y 72 meses, mediante la Escala Gross Motor Function Measure (GMFM). Usando solo la escala mental del Bayley Scales of Infant Development, BSID-II, Glenn, Cunningham y Dayus (2001) evaluaron 54 niños con SD con edades entre los seis y 24 meses. Son aún más escasos en estos niños los estudios de seguimiento de sus CD. El más referenciado, y quizás único, es el de Piper y colaboradores en 1986 publicaron los resultados de un estudio longitudinal del desarrollo realizado con Griffiths Developmental Scale en 32 niños con SD en sus primeros 
24 meses, registrando descensos de los valores iniciales (Piper, Gosselin, Gendron, \& Mazer, 1986). Los estudios de este tipo en América Latina no se conocen, por lo que no disponemos de valores de referencia en los diferentes periodos del desarrollo para una adecuada evaluación de estos niños.

En este artículo se da a conocer el perfil evolutivo de los niños con SD en las áreas de motricidad gruesa, motriz fina-adaptativa, audición y lenguaje, y el área personal y social, en sus primeros tres años de vida, logrados con un instrumento normalizado en Colombia como es la Escala Abrevada del Desarrollo EAD-1. Los valores de referencia servirán para identificar tempranamente las áreas más críticas, o de mayor necesidad de intervención, y evaluar el impacto de acciones terapéuticas, o programas de estimulación por agentes educativos y de salud. Con ello se buscará disminuir el impacto que su condición genética parece tenerles deparado a estos niños.

\section{Metodología}

Entre el periodo 2003-2012, se realizó a un estudio de cohorte de tipo prospectivo con un nivel descriptivo y correlacional. La población para este estudio estuvo representada por los niños del programa de síndrome de Down de Pediatría Social de la Universidad de Antioquia. La muestra no fue probabilística, sino intencional: el grupo de participantes estuvo conformado por 156 niños menores de 37 meses, quienes ya pertenecían o iban ingresando al programa: 81 hombres (52\%) y por 75 mujeres (48\%). Una tercera parte de los niños (32.8\%) fueron prematuros, y a quienes se hizo edad corregida los dos primeros años para las evaluaciones del desarrollo. En cuanto al tipo de síndrome, según 110 cariotipos evaluados, el 90\% tenía trisomía libre, translocación en el 5,5\%, y mosaísmo en un 4,5\%. Cerca del $83 \%$ de los niños era de estratos 1,2 y 3 o con mayor desventaja socio-económica, según el sistema de clasificación colombiana (SISBEN). Por ser un programa de universidad pública, no se consideró como criterio de selección el estrato socio-económico. Se tuvieron como criterios de exclusión, la coexistencia con el síndrome de parálisis cerebral y/o alguno de los trastornos del espectro autista.

\section{El instrumento}

Se utilizó la Escala Abreviada del Desarrollo (EAD-1). Esta prueba fue diseñada por el Ministerio de Salud de Colombia y el auspicio de Unicef para la evaluación y seguimiento del desarrollo de los niños entre 1 mes y 60 meses de edad, que asisten al programa de Crecimiento y Desarrollo en las instituciones de salud públicas y privadas. Según la Guía de Práctica Clínica basada en la evidencia para este programa, la EAD-1 tiene recomendación fuerte a favor para continuar utilizándola en la evaluación de los niños de todo el país (Ministerio de Salud y Protección Social, 2014). Este instrumento fue estandarizado en 16080 niños colombianos representativos de todas las regiones del país, y cuenta con estudios de validez y confiabilidad (Kuder-Richarson de 0.96). (Ministerio de Salud, 1993).

La EAD-1 está compuesta por 120 reactivos distribuidos en cuatro grupos de 30 ítems para cada una de las funciones que examina como son: la motricidad gruesa, motricidad fina, lenguaje y personal social (Ministerio de Salud, 1991).

La Motricidad Gruesa: hace referencia a la adquisición progresiva del control voluntario de los diferentes segmentos corporales en dirección cefalocaudal, proximal distal y organizados en posturas y movimientos dinámicos e integrados espacialmente.

La Motricidad Fina/adaptativa: hace referencia a la adquisición progresiva de habilidades manuales y digitales y el uso de instrumentos, con integración sensorial (táctil, visual, espacial), en la solución de problemas adaptativos.

La Audición y Lenguaje: hace referencia a la progresiva adquisición del lenguaje en los planos fonológico, morfológico sintáctico, semántico pragmático, manifiesto en la comprensión y expresión del código lingüístico. 
El área Personal y Social: hace referencia a la adquisición paulatina de patrones de intercambio social, autonomía e independencia y adaptación social.

Su tiempo de aplicación se estima entre 20-30 minutos. Cuenta con un sistema de registro y calificación de fácil manejo; las puntuaciones directas se transforman en categorías que permiten clasificar el desarrollo en un nivel de: Alerta, Medio, Medio-Alto y Alto (Ministerio de Salud, 1991). En el año 2001, la medición de los CD fue incorporada, en Pediatría Social por Rodríguez (2005), para el seguimiento de la población infantil con riesgo en el neurodesarrollo. En el caso de los niños prematuros, se corrige la edad cronológica con la edad gestacional de 40 semanas, hasta que cumplen los 24 meses de vida. La escala tiene el carácter de un instrumento de tamización y no de diagnóstico.

\section{El procedimiento}

A todos los niños del estudio se les elaboró una historia clínica de ingreso por pediatra. Los seguimientos del desarrollo se programaban cada tres meses y se realizaban de manera individual en sesiones diferentes a las consultas sobre el estado de salud. Entre el $75 \%$ y $80 \%$ de las evaluaciones fueron realizadas por el investigador principal, las demás fueron hechas por residentes de pediatría, practicantes de psicología y estudiantes de medicina, capacitados y supervisados directamente por el investigador principal. La edad de ingreso variaba entre los 2 y 24 meses. Cada sesión tuvo una duración promedio de 45 minutos distribuidos en la aplicación de la EAD-1, la explicación de los resultados, y recomendaciones a los padres del niño.

Para el tratamiento estadístico de los datos, se utilizó el software SPSS 15.0. Se usaron técnicas univariadas como medidas de tendencia central y de dispersión, para conocer el valor medio de los CD trimestral, semestral y general, su desviación estándar y la distribución normal y percentil. Para establecer las posibles diferencias en los CD entre cada una de las cuatro áreas evaluadas, se utilizaron técnicas bivariadas como prueba $t$ de Student de medias para muestras relacionadas (IC del 95\% y nivel de significación de $p>0.05$ ) o una prueba de rangos con signos de Wilcoxon de acuerdo al resultado de la prueba de normalidad de Kolmogorov-Smirnov.

\section{Resultados}

Entre los años 2003-2012, se estudió el Neurodesarrollo en 156 niños con SD, quienes asistieron a los controles del programa de Pediatría Social de la Universidad de Antioquia. En este período se realizaron 528 evaluaciones, con un promedio entre tres y cuatro valoraciones por paciente. Los niños que ingresaron más temprano al programa, tuvieron mayor cantidad de seguimientos, mientras los que eran mayores de 24 meses, o ingresaron al programa poco antes finalizar la investigación, alcanzaron el mínimo de una evaluación.

En un primer momento los CD obtenidos de las 528 evaluaciones realizadas a los 156 niños fueron sumadas en cada una de las áreas que examina la EAD-1 para obtener un promedio global en los primeros 36 meses de vida de estos niños, así como las medidas de dispersión, las que se pueden observar en la tabla 1 .

\section{TABLA 1}

Medidas de tendencia central y dispersión para las áreas evaluadas del desarrollo mediante la Escala Abreviada-1

\begin{tabular}{lcccccc}
\hline & & $\begin{array}{c}\text { Promedio } \\
\text { Motricidad } \\
\text { Gruesa }\end{array}$ & $\begin{array}{c}\text { Promedio } \\
\text { Motricidad } \\
\text { Fina }\end{array}$ & $\begin{array}{c}\text { Promedio } \\
\text { Audición y } \\
\text { Lenguaje }\end{array}$ & $\begin{array}{c}\text { Promedio } \\
\text { Personal y } \\
\text { Social }\end{array}$ & $\begin{array}{c}\text { Promedio } \\
\text { Global } \\
\text { Total }\end{array}$ \\
\hline Media & Válidos & 156 & 156 & 156 & 156 & 156 \\
Desv. típ. & & $\mathbf{6 2 . 7 6}$ & $\mathbf{6 5 . 4 1}$ & $\mathbf{6 4 . 4 8}$ & $\mathbf{6 8 . 4 6}$ & $\mathbf{6 4 . 2 3}$ \\
Asimetría & & 15.15 & 14.31 & 14.11 & 14.42 & 13.51 \\
Curtosis & & 0.58 & 0.26 & 0.41 & 0.1 & 0.62 \\
\hline Percentiles & 5 & $41.3 \mathrm{~b}$ & 0.24 & 0.28 & 0.15 & 0.67 \\
& 25 & 51.11 & $54.3 \mathrm{~b}$ & $43.3 \mathrm{~b}$ & $45.87 \mathrm{~b}$ & $46.1 \mathrm{~b}$ \\
& 50 & 62 & 65 & 54.37 & 58.55 & 55.35 \\
& 75 & 70.8 & 74.12 & 72.41 & 75.87 & 71.3 \\
& 95 & 93.4 & 91.6 & 91.6 & 98.68 & 91.4 \\
\hline
\end{tabular}

b Los percentiles se calcularán a partir de los datos agrupados. Fuente: elaboración propia.

Al promediar los $\mathrm{CD}$ de los primeros 36 meses de vida de las cuatro áreas estudiadas, 
se encontró un CD global de 64.23 con 13.51 puntos de desviación. Los $\mathrm{CD}$ en cada una de las cuatro áreas fueron los siguientes: en la motricidad gruesa, el valor promedio fue de 62.76 con una desviación estándar de 15.15 puntos, para la motricidad fina el valor medio hallado fue de 65.41 y una desviación estándar de 14.31, para el área de audición y lenguaje, se obtuvo una media de 64.48 y 14.11 puntos de desviación estándar, en cuanto al área personal y social se encontró un valor promedio de 68.46 y una desviación estándar de 14.42 puntos. Como puede apreciarse, las áreas de menor progresión son la motora gruesa y del lenguaje, mientras que el área personal y social presenta un mejor ascenso evolutivo. Los valores de curtosis y de asimetría para cada área evaluada con la EAD-1, permiten concluir que las puntuaciones tienen una distribución simétrica y normo-cúrtica, lo cual fue confirmado con la prueba de distribución normal de Kolmogorov-Smnirnov al tener una significancia $p>0.05$ (ver tabla 2).

\section{TABLA 2}

Prueba de distribución normal para los cocientes de desarrollo promediados a los 36 meses en las áreas evaluadas del desarrollo mediante la Escala Abreviada-1

\begin{tabular}{|c|c|c|c|c|c|}
\hline \multicolumn{2}{|c|}{ Estadísticos } & \multirow{2}{*}{$\begin{array}{c}\begin{array}{c}\text { Motricidad } \\
\text { Gruesa }\end{array} \\
156\end{array}$} & \multirow{2}{*}{$\begin{array}{c}\begin{array}{c}\text { Motricidad } \\
\text { Fina }\end{array} \\
156\end{array}$} & \multirow{2}{*}{$\begin{array}{c}\begin{array}{c}\text { Audición } \\
\text { Lenguaje }\end{array} \\
156\end{array}$} & \multirow{2}{*}{\begin{tabular}{|c|}
$\begin{array}{c}\text { Personal } \\
\text { Social }\end{array}$ \\
156
\end{tabular}} \\
\hline$N$ & & & & & \\
\hline \multirow{2}{*}{ Parámetros normales ${ }^{\mathrm{a}}$} & Media & 62.76 & 65.42 & 64.49 & 68.47 \\
\hline & Desviación típica & 15.15 & 14.31 & 14.11 & 14.43 \\
\hline \multicolumn{2}{|c|}{$Z$ de Kolmogorov-Smirnov } & 0.867 & 0.564 & 0.635 & 0.775 \\
\hline Sig. asintót. (bilateral) & & 0.44 & 0.908 & 0.815 & 0.585 \\
\hline
\end{tabular}

a La distribución de contraste es la Normal. Fuente: elaboración propia.

Ahora bien, para saber si esas diferencias en las puntuaciones halladas en las cuatro áreas o funciones eran estadísticamente significativas o no, se compararon los cocientes de cada área entre sí, mediante una prueba $t$ de medias para muestras relacionadas. $\mathrm{Al}$ respecto es posible afirmar, que existen diferencias significativas (intervalo de confianza del $95 \%$ y nivel de significación de $p<0.05)$ entre todos los promedios de las distintas áreas excepto entre la motricidad fina y la del lenguaje $(p=0.21)$. Estas diferencias entre varias de las funciones indican independencia entre ellas, y se retoman en la discusión (Ver tabla 3).

\section{TABLA 3}

Comparación de las medias de los $\mathrm{CD}$ de los 36 meses entre las cuatro áreas del desarrollo evaluadas

\begin{tabular}{lcccccc}
\hline \multicolumn{1}{c}{ Áreas de Comparación } & $\begin{array}{c}\text { Diferencia } \\
\text { de Media }\end{array}$ & $\begin{array}{c}\text { tip. } \\
\text { tipión }\end{array}$ & $t$ & $g l$ & $\begin{array}{c}\text { Sig. } \\
\text { (bilateral) }\end{array}$ \\
\hline Par 1 Motricidad Gruesa vs. Motricidad Fina & -2.65 & 8.35 & -3.96 & 155 & 0 \\
Par 2 Motricidad Gruesa vs. Audición / Lenguaje & -1.72 & 10.56 & -2.04 & 155 & 0.04 \\
Par 3 Motricidad Gruesa vs. Personal / Social & -5.7 & 9.84 & -7.23 & 155 & 0 \\
Par 4 Motricidad Fina vs. Audición / Lenguaje & 0.92 & 9.37 & 1.23 & 155 & 0.21 \\
Par 5 Motricidad Fina vs. Personal / Social & -3.04 & 8.15 & -4.66 & 155 & 0 \\
Par 6 Audición / Lenguaje vs. Personal/ Social & -3.97 & 8.01 & -6.19 & 155 & 0 \\
\hline
\end{tabular}

NotaPrueba $t$ de Student. (IC del 95\%

y nivel de significancia de $p>0.05$ )

Fuente: elaboración propia.

Para saber si los CD eran homogéneos durante los tres años de vida, se organizaron las puntuaciones por trimestres. De esta forma se obtuvo unos cocientes medios más ajustados al momento evolutivo por el que atraviesa todo niño. En la tabla 4, se presentan de manera resumida los valores encontrados en cada uno de los 12 trimestres en cada área, con su respectiva media y desviación estándar. El análisis por área da cuenta de resultados particulares. En la motricidad gruesa, se aprecia que inicia su desarrollo en un nivel alto, con un CD de 89.54 puntos, pero alrededor de los 36 meses, este cociente se ubica en un nivel medio de 56.79 puntos, es decir, hay una pérdida 33 puntos durante los primeros tres años de vida. La motricidad fina/adaptativa comienza igualmente en un nivel alto con un CD de 90.63 puntos y culmina a los 36 meses con un nivel de 59.81 puntos porcentuales. En el caso del lenguaje, se aprecia que comienza su evolución con el nivel más alto $(94.79 \%)$, y termina en el trimestre 12 con el nivel evolutivo más bajo (51.83\%). Es decir, hay una caída en 43 puntos porcentuales en los primeros 36 meses. Finalmente, en lo que se refiere al desarrollo del área personal y social por trimestres, se aprecia que inicia en un nivel alto con un CD de $90.88 \%$, y culmina a los 36 meses con el nivel más alto de las cuatro áreas evaluadas, un CD de 63.48\%.

Al graficar los $\mathrm{CD}$ por semestres, se puede evidenciar visualmente en la figura 1 este descenso de los cocientes en cada una de los 
campos o funciones examinadas. En ella se aprecia las curvas evolutivas obtenidas por los valores medios en cada semestre. Además, se destaca la caída de los CD en los dos primeros semestres para todas las funciones evaluadas por la EAD-1, y su estabilización posterior alrededor del tercer semestre de vida.

\section{TABLA 4}

Estadísticos descriptivos: media y desviación estándar (DE) de los cocientes de desarrollo para los primeros 12 trimestres de vida

\begin{tabular}{cllllllllll}
\hline \multirow{2}{*}{ Trimestre } & \multicolumn{2}{c}{$\begin{array}{c}\text { Motricidad } \\
\text { Gruesa }\end{array}$} & \multicolumn{2}{c}{ Motricidad Fina } & \multicolumn{2}{c}{$\begin{array}{c}\text { Audición y } \\
\text { Lenguaje }\end{array}$} & \multicolumn{2}{c}{$\begin{array}{c}\text { Personal y } \\
\text { Social }\end{array}$} & \multicolumn{2}{c}{ Global Total } \\
\cline { 2 - 12 } & \multicolumn{1}{c}{ Media } & DE & \multicolumn{1}{c}{ Media } & DE & Media & DE & \multicolumn{1}{c}{ Media } & DE & Media & DE \\
\cline { 2 - 12 } 1 & 89.54 & 16.19 & 90.63 & 17.26 & 94.79 & 10.89 & 90.58 & 14.22 & 90.88 & 13.21 \\
2 & 73.77 & 19.35 & 79.79 & 15.93 & 81.09 & 13.42 & 83.4 & 14.73 & 76.02 & 14.82 \\
3 & 73.83 & 14.48 & 74.4 & 13.53 & 75.9 & 13.88 & 77.6 & 13.63 & 72.7 & 12.48 \\
4 & 64.3 & 8.24 & 64.3 & 10.14 & 66.91 & 10.58 & 66.83 & 13.34 & 63.87 & 8.1 \\
5 & 57.35 & 8.41 & 61.15 & 11.32 & 64.76 & 13.64 & 64.71 & 12.68 & 59.4 & 10.24 \\
6 & 55.96 & 9.71 & 58.23 & 11.84 & 59.25 & 11.57 & 60.96 & 10.11 & 57.64 & 8.91 \\
7 & 53.84 & 9.31 & 58.58 & 13.41 & 58.86 & 9.56 & 62.19 & 14.84 & 58.02 & 10.8 \\
8 & 54.02 & 13.44 & 57.28 & 11.63 & 57.67 & 12.72 & 64.77 & 16.28 & 58.17 & 11.85 \\
9 & 53.97 & 15.46 & 55.18 & 14.73 & 54.74 & 13.52 & 62.05 & 15.98 & 56.64 & 13.59 \\
10 & 58.18 & 16.78 & 61.39 & 14.71 & 57.1 & 11.17 & 64.55 & 16.58 & 60.1 & 12.55 \\
11 & 58.23 & 14.4 & 61.44 & 13.49 & 52.79 & 10.7 & 63.18 & 12.7 & 60.13 & 10.88 \\
12 & 56.79 & 13.68 & 59.81 & 12.01 & 51.83 & 8.46 & 63.48 & 11.52 & 58.76 & 9.99 \\
\hline
\end{tabular}

Fuente: elaboración propia

Llama la atención que esta tendencia a un equilibrio relativo e incluso a una propensión a volver a ascender, se da en todas las áreas menos en la del lenguaje, ya que su propensión es a continuar cayendo. Este dato es de suma importancia para la comprensión del efecto posterior que el ámbito del lenguaje pueda promover en el desarrollo cognitivo y en la constitución del retardo mental o discapacidad intelectual.

Igualmente importante es el hallazgo de que entre los nueve y once meses de edad, las diferentes curvas evolutivas descienden o superan el umbral de los 75-70 puntos, es decir atraviesan el límite entre un desarrollo normal $(115-86)$ y un retardo madurativo (85-70,) a una zona de retardo patológico (69 -5). En otras palabras, se puede afirmar que durante los primeros 10 meses de vida, la mayoría de los niños con SD pueden tener niveles de desarrollo dentro de los límites medio y bajo, similares a los de niños con desarrollo típico, pero que inexorablemente al aproximarse al primer año de vida su desarrollo empiezan a entrar o caer en la zona de retardo evolutivo.

\section{Figura 1}

Curvas de desarrollo para los primeros 36 meses de vida, en las cuatro áreas evaluadas por la EAD-1, con punto de referencia el promedio semestral.
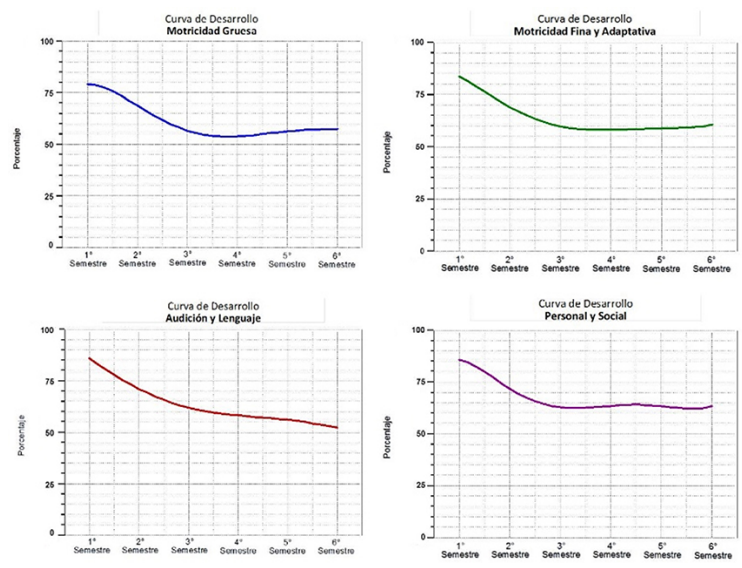

Fuente: elaboración propia.

\section{Discusión}

Un primer punto para tener en cuenta en la discusión, es el hecho de haber obtenido los cocientes de desarrollo en los niños con SD en sus primeros tres años, con un instrumento estandarizado en población colombiana, lo que nos permite tener valores de referencia para la evaluación y asesoría al proceso evolutivo de estos niños. Un segundo punto, quizás el más importante, es haber conseguido evaluaciones en diferentes momentos del curso de la vida de los niños, lo que permitió construir curvas diferenciales de desarrollo, al menos no conocidas antes, lo que permite tener un mayor conocimiento de su proceso evolutivo y tomar medidas terapéuticas de manera más oportuna y mejor dirigidas.

En cuanto al primer punto, se puede decir que el cociente global medio de 64.2 (13.5) encontrado en este estudio no difiere de manera notable de aquel estimado por el instrumento más referenciado mundialmente: la Escala de Desarrollo Bayley (Bayley Scales of Infant Development, BSID). En un estudio realizado en Inglaterra por Glenn et al., (2001), en el que se compararon las dos versiones de la escala mental del Bayley (BSID y BSID-II), el 
cociente o índices de desarrollo mental (IDM) medio alcanzados por los niños con SD con la BSID-II, fue de $63.9(D E=17.3)$ frente a un cociente de $72.3(D E=16.1)$ obtenido con la escala de Bayley original de 1969. El estudio fue transversal y contó con una muestra de 54 niños con SD, con edades entre 7 y 43 meses, y una de 20 niños con desarrollo típico de 6 a 24 meses de edad. Los valores logrados por este último estudio con la BSID-II son muy similares a los obtenidos con la EAD-1. En efecto, los promedios de los cocientes alcanzados por los niños al llegar a los 36 meses de vida fueron: para la motricidad gruesa, de 62.7 puntos; para la motricidad fina, de 65.4 puntos; para el lenguaje, de 64.4 puntos, y para el área personal y social, de 68.4 puntos porcentuales. En un estudio más reciente, Ellis y colaboradores (2008) realizaron un ensayo clínico controlado aleatorizado con 156 niños menores de 7 meses con SD, para evaluar mediante la escala de Griffiths los efectos en el desarrollo del uso de antioxidantes y ácido fólico después de 18 meses de tratamiento. Los CD que encontraron a las edades de 18-25 meses estaban entre 56.9 y 58 , que son prácticamente los mismos que se hallaron en el $6^{\circ}, 7^{\circ}$ y $8^{\circ}$ trimestre con la EAD-1 (ver tabla 2).

Por otro lado, se pudo apreciar en nuestro estudio que las áreas de menor avance fueron la motora gruesa y especialmente el área del lenguaje, mientras que el área personal y social presentaron un mayor ascenso evolutivo. Este patrón es similar al del estudio de Chen y Woolley en 1978, citado por Palisano et al. (2001), en el que evaluaron 106 niños con SD mediante el Denver Developmental Screening Test. Los CD que ellos lograron obtener fueron: 48 puntos para el dominio del lenguaje, 55 para motricidad gruesa, 62 para motricidad fina-adaptativa y 65 para el dominio personal-social. Si bien el orden y las áreas comprometidas corresponden a las mismas halladas en el presente estudio, la discrepancia en los valores puede deberse al rango más amplio de edad (hasta los 96 meses) estudiado por estos autores.

Esta regularidad evolutiva también fue observada por Piper et al. (1986), utilizando el test The Griffiths Developmental Scale. El estudio, que incluyó a 32 niños con SD durante los dos primeros años de vida, mostró que el mayor grado de retraso se ubicaba en el área de audición y lenguaje, seguido por el dominio motor, el cual experimentó el descenso más grave a los 24 meses. A la edad de seis meses, el cociente de desarrollo motor era de 87 puntos y de 75 para el dominio de audición y habla; los que descendieron al nivel de 59 y 56 respectivamente, a la edad de los dos años. Al parecer, el dominio menos afectado a esta edad era el personal-social, que alcanzó una puntuación de 68.

En cuanto al segundo punto, y quizás el más importante logro que emerge de nuestro estudio, corresponde a la obtención de CD para diferentes momentos de los primeros años de vida y la identificación de un patrón de variación de esos cocientes en función del tiempo. El desconocimiento de estas variaciones o descenso esperable en la curva evolutiva, pueden llevar a abandonar programas terapéuticos $\mathrm{o}$ de estimulación al no ver aumento de los cocientes, sino valores iguales o pequeñas variaciones con respecto a los cocientes antes de iniciar la intervención. Por ejemplo, un niño con SD que a los seis meses tiene un $\mathrm{CD}$ de $75 \%$ y luego de una intervención estructurada, a los 24 meses alcanzaba un CD de $70 \%$, el programa podía ser considerado poco o nada efectivo cuando en realidad los cocientes esperados serian de $56 \%$ a esa edad.

El análisis de las variaciones de los cocientes permitió dar cuenta de tres momentos diferentes, en la organización de las funciones mentales básicas durante los primeros tres años de vida (ver figura 2). Este hallazgo se alcanzó en virtud de haber ordenado las puntuaciones en trimestres, agrupación que permitió construir una curva de desarrollo en la que se evidencian los rasgos distintivos con los que delimitan un fenotipo evolutivo. Para poder comprender mejor el peso de los $\mathrm{CD}$, es necesario tener en cuenta la tabla de clasificación del desarrollo de Bianchi (Rodríguez, 2005) en la que se establece: un nivel normal de desarrollo cuando los CD se ubican entre 114-85 puntos, un retardo madurativo cuando los CD están entre 84-70, y un retardo 
patológico cuando los $\mathrm{CD}$ son inferiores a 70 puntos.

El primer momento de la curva abarca los primeros nueve meses de vida, donde el desarrollo de la mayoría de los niños con SD, exceptuando los que presentan hipotonía severa y/o cardiopatías graves, tienen características y ritmos similares a la de niños sin esta condición genética. Es decir, los CD iniciales en las áreas motoras gruesa, adaptativa, del lenguaje y la personal social arrancan desde un nivel medio y medio bajo $(C D=100-86)$ y prosigue por un nivel de un retraso madurativo $(\mathrm{CD}=$ 85-70). Por lo tanto, no es extraño que durante los primeros trimestres de vida de un niño con SD, en buenas condiciones de salud, con adecuada ganancia de talla y peso, una apropiada estimulación y vinculación afectiva, tenga niveles de desarrollo similares y cercanos a los niños sin SD.

\section{Figura 2}

Curva de desarrollo de la motricidad gruesa (MG) para los primeros para los primeros 12 trimestres de vida

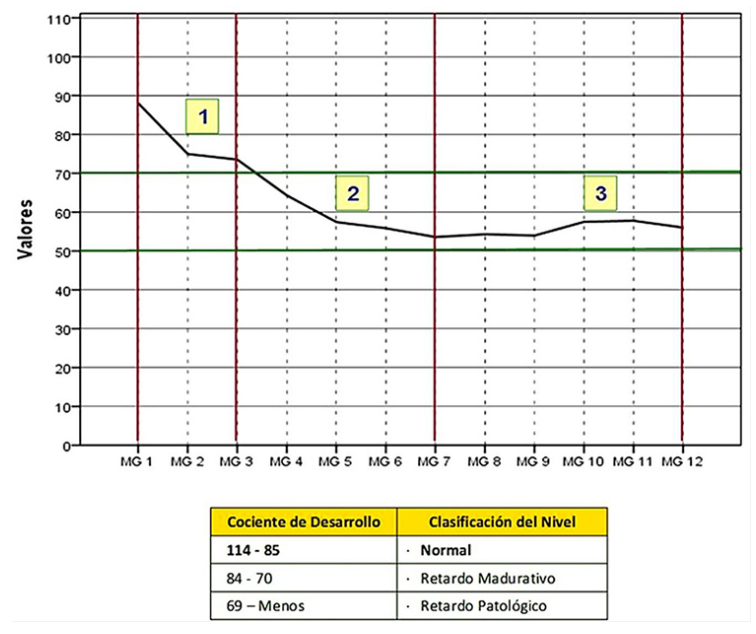

Fuente: elaboración propia.

Quizás estas sean condiciones esenciales, aunque no suficientes, para mantener el desarrollo cerebral. Según Nadel (1999), un amplio grupo de estudios sostienen que al momento del nacimiento, el cerebro de un bebé con SD es indistinguible en muchos aspectos al de otro niño sin esta afectación, pero a partir de los 6 meses de edad, una serie de diferencias importantes empiezan a evidenciarse. Las condiciones neuropatológicas después de 3-5 meses de edad postnatal incluyen: un acortamiento de la longitud frontooccipital del cerebro como consecuencia, al parecer, de una reducción en el crecimiento de los lóbulos frontales, un estrechamiento de la circunvolución temporal superior, una disminución del tamaño del tallo cerebral y del cerebelo, y una reducción del $20-50 \%$ en el número de neuronas corticales granulares Fidler y Nadel (2007). Si bien en los niños con SD puede existir un retraso en la mielinización, un 75\% de los ellos está dentro del rango normal durante el desarrollo temprano.

Entonces, icómo podría explicarse este nivel de desarrollo inicial en los niños con SD durante los primeros trimestres de vida? Si bien no hay una repuesta clara para este interrogante, a modo de hipótesis es posible plantear que los ítems de las escalas que aprueban los niños en estos primeros meses, corresponden más a protoconductas, es decir, reacciones que están en la transición de lo innato a lo aprendido. Estos comportamientos se apoyan en acciones que tienen un alto contenido de programación genética, de patrones madurativos, de actividad espontánea y, en menor grado, de lo que Azcoaga $(1982,1983)$ denomina "aprendizaje fisiológico", el cual es la base para la organización de las praxias, las gnosia y lenguaje. Ejemplos de esas protoconductas son: el balbuceo, el juego vocal, la búsqueda de fuentes sonoras, el seguimiento de objetos, el asir y exploración oral, el giro del tronco, el sostén cefálico, entre otros. Estas conductas que se examinan en estos primeros meses, al estar en este rango transicional entre lo programado y lo adquirido, pueden tener un mejor rendimiento psicométrico en estos niños. Pero cuando las conductas que se evalúan empiezan a depender de procesos de aprendizaje fisiológico, se registra un menor resultado en los CD.

El segundo momento, comprende el período entre los 10 y 18 meses de vida. Se caracteriza por que los $\mathrm{CD}$ de los niños con SD caen por debajo de los 70 puntos. Es decir, su rendimiento pasa de una zona de desarrollo 
medio bajo o con retardo madurativo, a la zona de retardo patológico $(\mathrm{CD}<70)$. La caída en los cocientes se debe a la creciente y ahora evidente dificultad para adquirir ciertas destrezas que podrían denominarse "organizadores filogenéticos" de primer orden. Estos organizadores filogenéticos, que representan hitos en el proceso de hominización, corresponden en una escala de desarrollo ontogenético a: la bipedestación en la motricidad gruesa; la solución del conflicto de manipular un tercer objeto cuando ambas manos están ocupadas en la motricidad fina-adaptativa; el pasaje del nivel prelingüístico al lingüístico, es decir, con la aparición de la palabra articulada en el área del lenguaje; y en el área social a la aparición de los actos protoimperativos, de atención conjunta. Estos retos evolutivos exigen no solo una continuidad en el ritmo de la mielinización, sino la disponibilidad cerebral de una mayor organización estructural y funcional, y no solo de una continuidad en el ritmo en la mielinización. Nadel (1999) plantea que hay evidencias de que algunos cambios en el SNC a los 6 meses de vida ya son evidentes. Este autor plantea una reducción en el crecimiento de los lóbulos frontales, un estrechamiento del giro temporal superior, la disminución del tamaño del cerebelo y una reducción significativa en el número de neuronas corticales granulares.

Pero además, estos desafíos evolutivos exigen la aparición de cambios que, en términos de Overton (2003), expresen modificaciones de tipo transformacional y variacional. Los cambios transformacionales son cambios cualitativos y discontinuos, son modificaciones en la forma, en la organización o en la estructura de cualquier sistema. Esto implica que a medida que cambian las formas, se incrementa la complejidad de los componentes de manera no lineal, ni aditiva. Por otra parte, los cambios variacionales son de tipo cuantitativo y continuo, y se refieren al grado en que un cambio varía dentro de la norma, o promedio, reflejando mayor precisión o exactitud. Por lo observado en la investigación, los niños con SD muestran las mayores dificultades en la emergencia de los cambios transformacionales y, en menor medida, en los variacionales, los cuales avanzan lentamente pero solo hasta que se den los primeros. Los organizadores filogenéticos realizados en el curso de la ontogenia, corresponderían a lo transformacional en tanto representan modificaciones de la estructura y función. Pasar de desplazarse de cuatro puntos de apoyo a dos, del gesto a la significación y representación mental y de la simple manipulación a la instrumentación y solución de problemas, son cambios transformacionales que el niño con SD debe afrontar para lograr el primer gran salto cognitivo y la acumulación de experiencias de tipo variacional, hasta que un nuevo nivel o exigencia transformacional tenga lugar.

Por último, el tercer momento identificado está constituido por el período que va desde los 18 a 36 meses de vida. En este lapso, los CD tienden a desacelerar su caída y a estabilizarse alrededor de valores que oscilan entre los 55 y 64 puntos porcentuales. Una explicación hipotética para esta estabilización temporal de las curvas, teniendo en cuenta los tipos de cambios evolutivos planteados por Overton (2003), sería que alrededor de los 18 a 21 meses de vida los niños alcanzan los cambios transformacionales u organizadores filogenéticos de primer orden (propuesto en este estudio) que no habían logrado conseguir previamente. Ahora lo que continúa para ellos es la acumulación de cambios variacionales (cuantitativos) hasta la aparición de un nuevo ordenador evolutivo o transformacional. En efecto, al hacer la conversión de los cocientes estimados para los 18-21 meses de edad cronológica, el resultado muestra que su edad madurativa corresponde a 11-12 meses, que es el período en que la mayoría de los niños sin esta condición genética, están alcanzando los hitos u "organizadores filogenéticos".

Aunque la tendencia en este tercer momento es alcanzar una estabilidad relativa, e incluso a un ligero ascenso en los CD en la mayoría de las áreas o funciones que se están organizando, en el área del lenguaje la propensión de su CD es a continuar decreciendo. Mientras en los primeros 24 meses el menor ascenso es para la función motora, esta diferencia se invierte a partir de los dos años, tras los que el lenguaje queda 
con un menor rendimiento o con un cociente decreciente, lo que inexorablemente se refleja en las deficiencias cognitivas posteriores.

La mayor preocupación de los padres de estos niños durante los primeros 36 meses es el desarrollo de la motricidad gruesa, particularmente en cuanto a la marcha autónoma. De ello dan cuenta múltiples investigaciones dirigidas al estudio de reacciones posturales (Haley, 1986), la estimulación de la marcha con el uso de bandas rodantes (Ulrich, Ulrich, Angulo-Kinzler, \& Yun, 2001), y el uso de ortesis para favorecer la bipedestación (Selby-silverstein, Hillstrom, \& Palisano, 2001). Alrededor de los dos a tres años, cuando los niños con SD ya han logrado caminar de modo independiente, las inquietudes de los padres se dirigen, ahora, al retraso lingüístico que presentan, habiendo perdido un tiempo valioso relacionado con el periodo crítico de neurodesarrollo para la estimulación e intervención de esta función (Rodríguez, 2005).

Las habilidades de producción del lenguaje frecuentemente están más retrasadas que las habilidades de comprensión del lenguaje (Capone, 2004; Ypsilanti, \& Grouios, 2008). El aprendizaje de las primeras diez palabras, según Oliver y Buckley (1994), sucede entre los 19 y 38 meses de vida y en más de la mitad de los niños con este síndrome no se observa la "explosión de vocabulario", que se describe a los 24 meses de edad en niños con desarrollo normal. La edad media para la consolidación de la frase de dos palabras en los niños con SD es 36.9 meses, lo que sugiere un retraso de alrededor de 18 meses. Este retraso en el lenguaje expresivo se vuelve aún más pronunciado a medida que progresa la complejidad de la sintaxis, y el vocabulario se amplía cuantitativa y cualitativamente. Es probable que la hipofrontalidad del neocórtex, frecuentemente reportado en el SD, junto con una reducción en las proyecciones frontales del cuerpo calloso y con el compromiso cerebeloso, se relacionen de manera significativa con la baja fluidez verbal, la inteligibilidad del habla y las dificultades con los aspectos formales de las funciones del lenguaje (Rondal, 1995). Pero también, como sostienen Marder y Nicholmain (2006), algunos niños con SD pueden tener dificultades con la formas sintácticas y fonológicas, o con el acceso a los contenidos semánticos, estos últimos asociados con un menor desarrollo del lóbulo temporal y el hipocampo.

Hay que anotar que la discapacidad intelectual en estos niños no solo es resultado del efecto de una sola función psicológica superior alterada. Sin embargo, el lenguaje juega un papel preponderante en su génesis. Esto devela la imperiosa necesidad de intervenir de manera prioritaria esta función psicológica superior, y poder mitigar, en un algún grado, la discapacidad posterior. Los niños que tienen un buen desarrollo lingüístico pueden acceder más fácilmente a los retos de la escolarización: el aprendizaje de la lectura, la escritura y las matemáticas, lo que potencializa sus posibilidades cognitivas futuras y calidad de vida.

\section{Conclusiones}

Con esta investigación de tipo longitudinal se logra, no solo reconocer la Escala Abreviada del Desarrollo EAD-1 como un instrumento útil y válido para evaluar a los niños con $\mathrm{SD}$, sino también suplir la falta de valores de referencia (medias, desviaciones típicas y percentiles), por trimestres y curvas en las que se grafica el curso del desarrollo diferencial durante los primeros 36 meses de vida. Los resultados aquí encontrados aportan al seguimiento del neurodesarrollo de un niño con SD, a la orientación y asesoría a padres y terapeutas, al poder estimar si su desarrollo avanza o no en sus diferentes momentos evolutivos, de acuerdo con lo esperado para su edad y para su condición genética. Vale la pena recordar que hace casi 30 años Sharav, Collins, \& Shlomo (1985) afirmaron que solo a través de mediciones repetidas de la misma persona, pueden ser descubiertos los patrones individuales, así como el desarrollo del grupo; que la creación de las tablas percentiles permitiría las predicciones del modelo de desarrollo intelectual futuro, así como en la evaluación sobre el éxito del uso de tratamientos nuevos o controvertidos 
para los niños con SD. Precisamente esta es la proyección de esta investigación a futuro; identificar los factores que están asociados a su proceso evolutivo, así como el efecto de intervenciones puntuales en las variaciones de los cocientes de desarrollo, especialmente en el área del lenguaje, asociada a la mayor discapacidad a futuro en estos niños.

\section{Limitaciones}

Las principales limitaciones que afectaron la construcción de una base de datos con mayor información y precisión, fueron: no todos los niños ingresaron en el primer trimestre de vida; el 63\% ingresó después de los seis meses de vida, lo que afectaba el número de evaluaciones en los primeros meses de vida. Por otro lado, los frecuentes problemas de salud de los niños, las precarias condiciones económicas de las madres, las dificultades para salir del barrio por violencia afectaban la asistencia esperada de la madre y el niño a las citas de control, teniendo que postergar y reprogramar los seguimientos.

\section{Consideraciones éticas}

Para esta investigación, se tuvieron en cuenta las disposiciones contempladas en la resolución 008430 de 1993, del Ministerio de Salud de Colombia, específicamente en el título II (de la investigación en seres humanos), Capítulo 1 (De los aspectos éticos de la investigación en seres humanos). Algunas de ellas son las siguientes:

Artículo 5. En toda investigación en la que el ser humano sea sujeto de estudio, deberá prevalecer el criterio del respeto a su dignidad y la protección de sus derechos y su bienestar.

Artículo 6. La investigación que se realice en seres humanos se deberá desarrollar conforme a los siguientes criterios:

a) Se ajustará a los principios científicos y éticos que la justifiquen.

b) Deberá prevalecer la seguridad de los beneficiarios, y expresar claramente los riesgos (mínimos). c) Contará con el Consentimiento Informado y por escrito del sujeto de investigación o su representante legal con las excepciones dispuestas en la presente resolución.

d) Deberá ser realizada por profesionales con conocimiento y experiencia para cuidar la integridad del ser humano bajo la responsabilidad de una entidad de salud, supervisada por las autoridades de salud, siempre y cuando cuenten con los recursos humanos y materiales necesarios que garanticen el bienestar del sujeto de investigación.

Artículo 8. En las investigaciones en seres humanos se protegerá la privacidad del individuo, sujeto de investigación, identificándolo solo cuando los resultados lo requieran y este lo autorice.

Artículo 11. En el que se estipula la clasificación de las investigaciones. El presente estudio se ubica en la siguiente categoría: a) Investigación sin riesgo: Son estudios que emplean técnicas y métodos de investigación documental retrospectivos y aquellos en los que no se realiza ninguna intervención o modificación intencionada de las variables biológicas, fisiológicas, sicológicas o sociales de los individuos que participan en el estudio, entre los que se consideran: revisión de historias clínicas, entrevistas, cuestionarios y otros en los que no se le identifique ni se traten aspectos sensitivos de su conducta (Ministerio de Salud, 1993, p. 1, 2).

\section{Declaración de conflictos de intereses}

Los autores declaran no tener conflictos de intereses por presentar vínculos con entidades financieras, farmacéuticas, políticas o religiosas que pudieran afectar el resultado de esta investigación. Este trabajo fue producto de los tiempos designados para investigación en el plan de trabajo docente de Pediatría Social de la Facultad de Medicina de la Universidad de Antioquia, desde 2003 y culminado durante la 
realización de la Maestría en Psicología de la misma Universidad en el año 2012.

\section{Agradecimientos}

Agradecimiento a los niños y niñas con síndrome de Down y sus padres, la Asociación ASIDO CON AMOR, ANDECOL, a los profesores y personal enfermería de Pediatría Social y especialmente a la profesora Luz Elena Gómez Londoño.

\section{Referencias}

Abbeduto, L., Warren, S. F., \& Conners, F. A. (2007). Language development in Down syndrome: from the prelinguistic period to the acquisition of literacy. Mental Retardation and Developmental Disabilities Research Reviews, 13, 247 - 261.

Antonarakis, S. E., Lyle, R., Chrast, R., \& Scott, H. S. (2001). Differential gene expression studies to explore the molecular pathophysiology of Down syndrome. Brain research. Brain research reviews, 36(2-3), 265-74. Disponible en: http://www.ncbi.nl m.nih.gov/pubmed/11690624.

Artigas, M. (2004). Síndrome de Down (Trisomia 21), Protocolos de la Asociación Española de Pediatría [consultado 15/11/2009]. Disponible en: http://www.aeped.es/sites/d efault/files/documentos/6-down.pdf

Azcoaga, J. E. (1982). Fisiopatología de la Actividad Nerviosa Superior en la Deficiencia Mental. Revista de la Asociación Argentina para el Estudio Científico de la Deficiencia Mental, 2, 9-24.

Azcoaga, J. E. (1983). Las funciones cerebrales superiores y sus alteraciones en el niño y en el adulto. Buenos Aires, Argentina: Paidós.

Capone, G. T. (2004). Down Syndrome: Genetic insights and thoughts on early intervention. Infant and Young Children, 17(1), 45-58.

Colombia, Ministerio de Salud. (1991). Escala Abreviada del Desarrollo: manual de instrucciones. Santa Fé de Bogotá.
Colombia, Ministerio de Salud. (1993). Diseño y Normalización de la Escala Abreviada del Desarrollo: Informe técnico. Santa Fé de Bogotá.

Colombia, Ministerio de Salud y Protección Social. (2014). Gui\#a de Pra\#ctica Cli\#nica (GPC) Basada en la evidencia para la promoción del crecimiento, detección temprana y enfoque inicial de alteraciones del crecimiento en niños menores de 10 años y la promoción del desarrollo, detección temprana y enfoque inicial de las alteraciones del desarrollo en niños menores de 5 años en Colombia. Disponible en: http://gpc.minsalud.gov.co/gpc_sites/R epositorio/Conv_563/GPC_crecimiento/G uia_Completa_C_D.pdf

Ellis, J. M., Tan, H. K., Gilbert, R. E., Muller, D. P., Henley, W., Moy, R., \& Green, H. (2008). Supplementation with antioxidants and folinic acid for children with Down's syndrome: randomised controlled trial. British Medical Journal, 336(7644), 594-597.

Fidler, D. J., \& Nadel, L. (2007). Education and children with down syndrome: neuroscience, development, and intervention. Human Development, 13, 262 - 271. http://doi.org/10.1002/mrdd.

Flórez, J. (1991). Patología Cerebral y Aprendizaje en el síndrome de Down. En: El sindrome de Down y Educación. Barcelona. Ed. Salvat.

Glenn, S. M., Cunningham, C. C., \& Dayus, B. (2001). Comparison of the 1969 and 1993 standardizations of the Bayley Mental Scales of Infant Development for infants with Down syndrome. Journal of Intellectual Disability Research, 45, 56-62.

Haley, S. M. (1986). Postural Reactions in Infants with Down Syndrome. Physical Therapy, 66(1), 17-22.

Hayes, A., \& Batshaw, L. (1993). Síndrome de Down. En: Clínicas Pediátricas de Norteamérica. México. Ed. Interamericana Mc Graw Hill.

Marder, L., \& Nicholmain, C. (2006). Promoting language development for children with 
Down's syndrome. Current Paediatrics, 16(7), 495-500. http://doi.org/10.1016/ j.cupe.2006.08.022

Martin, G. E., Klusek, J., Estigarribia, B., \& Roberts, J. E. (2005). Language Characteristics of Individuals with Down Syndrome. Science, 309(5735), 696b-696b.

Moore, D. G., Goodwin, J. E., \& Oates, J. M. (2008). A modifed version of the Bayley Scales of Infant DevelopmentII for cognitive matching of infants with and without Down syndrome. Journal of Intellectual Disability Research, 52 (june), 554-561. http://doi.org/10.1111/ j.1365-2788.2008.01064.x.

Nadel, L. (1999). Down syndrome in Cognitive Neuroscience Perspective. In Tager-Flusberg H (Ed.), Neurodevelopmental Disorders (pp. 197-223). Cambridge: MIT Press.

Oliver, B., \& Buckley, S. (1994). The language development of children with Down's syndrome: First words to two-word phrases. Down Syndrome Research and Practice, 2(2), 71-75.

Overton, W.F. (2003). Development Across the Life Span: philosophy, concepts, theory. In: R. M. Lerner, M A. Easterbrooks, \& J. Mistry (Eds.) Comprehensive Handbook of Psychology: Developmental Psychology, 6. (pp. 13-42). New York: Wiley.

Palisano, R. J., Walter, S. D., Russell, D. J., Rosenbaum, P. L., Gémus, M., Galuppi, B. E., \& Cunnigham, L. (2001). Gross Motor Function of Children with Down Syndrome: Creation of Motor Growth Curves. Arch Phys Med Rehabil, 82, 494-500.

Pinter, J. D., Eliez, S., Schmitt, J. E., Capone, G. T., \& Reiss, A. L. (2001). Neuroanatomy of Down's syndrome: a high-resolution MRI study. American Journal of Psychiatry, 158(10), 1659-1665.

Piper, M. C., Gosselin, C., Gendron, M., \& Mazer, B. (1986). Developmental profile of Down's syndrome infants receiving early intervention. Child Care, Health and Development, 12, 183-194
Rodríguez, J.C. (2012). El perfil evolutivo y los factores asociados al desarrollo de los niños con síndrome de Down en sus primeros tres años de vida. Tesis de grado para optar por el título de: Magister en psicología. Universidad de Antioquia, Medellín.

Rodríguez, J.C. (2005). Guía de Atención Integral en Salud para Niños con Sindrome de Down. Monografía para optar por el título de: Especialista en Niños con Énfasis en Psicología Clínica Comportamental y Neuropsicología Infantil. Universidad de Antioquia, Medellín.

Rondal, J.A. (1995), Exceptional language development in Down syndrome. Implications for the cognition-language relationships, New York: Cambridge University Press.

Selby-silverstein, L., Hillstrom, H. J., \& Palisano, R. J. (2001). The effect of foot orthoses on standing foot posture and gait of young children with Down Syndrome. NeuroRehabilitation, 16, 183-193.

Sharav, T., Collins, R., \& Shlomo, L. (1985). Effect of maternal education of prognosis of the developmental in SD.pdf. Pediatrics, 76(3), 387-391.

Ulrich, D. A., Ulrich, B. D., Angulo-Kinzler, R., \& Yun, J. (2001). Treadmill Training of Infants with Down Syndrome: EvidenceBased Developmental Outcomes. Pediatrics, 108(5). http://doi.org/10.1542/ peds.108.5.e84.

Ypsilanti, A., \& Grouios, G. (2008). Linguistic profile of individuals with Down syndrome: comparing the linguistic performance of three developmental disorders. Child Neuropsychology, 14, 148-170.

\section{Notas}

* Artículo de investigación. 